\title{
Efecto de las heterogeneidades de textura sobre el comportamiento mecánico de una aleación superplástica de Al-6\% Cu-0,4\% Zr
}

\author{
M. Eddahbi* y O.A. Ruano*
}

\begin{abstract}
Resumen Se ha estudiado el comportamiento mecánico y microestructural de una aleación superplástica de $\mathrm{Al}-6 \% \mathrm{Cu}-0,4 \% \mathrm{Z}$ a $480^{\circ} \mathrm{C} / 5 \cdot 10^{-4} \mathrm{~s}^{-1}$. La microestructura en estado de recepción consta de una subestructura fina. Presenta gradientes de textura a través del espesor de la chapa. En la superficie, la textura esta compuesta, principalmente, por la orientación $\{311\}<233>$ mientras que en el centro por la orientación $\{631\}<113>$. La curva de tracción (s)-(e) presenta tres estados de deformación, llamados estados I, II y III. En el estado I la textura inicial aumenta ligeramente. En el estado II la intensidad de la orientación $\{311\}<233>$ disminuye, lo que sugiere la operación de un mecanismo de deformación por deslizamiento de fronteras de grano. Sin embargo, en el centro, la orientación $\{631\}<113>$ se transforma en la orientación $\{110\}<111>$ por un proceso de deslizamiento de dislocaciones. Este comportamiento prosigue durante el estado III en el cual la orientación $\{631\}<113>$ desaparece totalmente.
\end{abstract}

Palabras clave Superplasticidad. Microestructura. Gradientes de textura.

\section{Effect of inhomogeneity of texture on mechanical behaviour of an $\mathrm{Al}-6 \% \mathrm{Cu}-0.4 \% \mathrm{Zr}$ superplastic alloy}

\begin{abstract}
Mechanical and microstructural characterization was carried out at $480{ }^{\circ} \mathrm{C} / 5 \cdot 10^{-4} \mathrm{~s}^{-1}$ on an $\mathrm{Al}-6 \% \mathrm{Cu}-0.4 \% \mathrm{Zr}$ superplastic alloy. The as-received material showed a fine deformed substructure. A texture gradient was present through the thickness of the rolled sheet. At the surface, the texture found was close to the orientation $\{311\}<233>$ whereas in the midplane of the sheet it was $\{631\}<113>$. The (s)-(e) tensile test curve presented three deformation stages called, stage I, II and III. The global texture in stage I increased slightly. In stage II, the intensity of orientation $\{311\}<233>$ decreased, suggesting that a grain boundary sliding deformation process should take place near the surface. However, in the center of the sheet, the $\{631\}<113>$ orientation transformed to the orientation $\{110\}<111>$ by slip process. This behaviour continued in the stage III, where the orientation $\{631\}<113>$ totally disappeared.
\end{abstract}

Keywords Superplasticity. Microstructure. Texture gradients.

\section{INTRODUCCIÓN}

El objetivo de la mejora de las propiedades de los materiales ha llevado a profundizar y establecer las correlaciones entre los parámetros que determinan la microestructura del material antes del conformado (estado inicial o de partida) y la microestructura resultante del mismo (estado final). Asimismo, ha llevado a determinar los mecanismos de deformación que entran juego, ya que el control de estos parámetros determina las propiedades del material.
La anisotropía de las propiedades mecánicas observada en ciertas aleaciones de aluminio ha sido atribuida a la presencia de heterogeneidades de textura a través del espesor de la chapa de laminación, producido por el procesado ${ }^{[1-3]}$. Se ha observado que el límite elástico y la resistencia a la tracción hallados en el centro de la chapa son elevados en comparación con aquellos correspondientes a toda la chapa ${ }^{[3]}$. Asimismo, se ha revelado, en algunas aleaciones superplásticas de aluminio, que el aspecto inicial de la microestructura, granos

${ }^{*}$ ) Departamento de Metalurgia Física, Centro Nacional de Investigaciones Metalúrgicas, CSIC, Avda. Gregorio del Amo, 8. 28040 Madrid (España). 
alargados con la orientación $\{110\}<112>$ en el centro y equiáxicos y orientados hacia (001)[110] en la superficie, permanece aún después del 100\% de deformación superplástica, DSP ${ }^{[4]}$. La alta sensibilidad a la velocidad de deformación determinada $(\mathrm{m}=0,5)$ y la disminución de la textura sugiere que el deslizamiento de fronteras de grano, DFG, es el mecanismo responsable de la deformación. Sin embargo, las observaciones microestructurales descartaron dicho mecanismo. Los gradientes de microestructura (textura y estructura de grano), por tanto, influyen sobre el comportamiento superplástico del material y las propiedades mecánicas.

Las aleaciones del sistema $\mathrm{Al}-\mathrm{Cu}-\mathrm{Zr}$ se utilizan en la conformación superplástica tanto en el campo aeronáutico como de locomoción. Para mejorar aún las propiedades de estas aleaciones en particular y de los materiales superplásticos en general, se llevaron a cabo numerosos trabajos sobre los aspectos tanto macro y microscópicos de la deformación superplástica ${ }^{[6}$ y 7$]$. Por ejemplo, el estudio de la anisotropía en estas aleaciones ha demostrado que las muestras ensayadas longitudinalmente y transversalmente mostraron el mismo comportamiento mecánico, lo que ha sugerido que el mecanismo en ambos casos posee la misma naturaleza ${ }^{[8]}$. No obstante, en estos estudios se ha ignorado por completo la presencia de gradientes de textura en el material de partida y por consiguiente el efecto que pueda tener sobre el comportamiento superplástico de dichas aleaciones.

En este trabajo, se determinarán, en primer lugar, los gradientes de textura a través del espesor de una chapa de laminación de la aleación Al-6\%$0,4 \% \mathrm{Cu}$, en su estado de recepción. Por otro lado, se determinarán en diferentes zonas del espesor los cambios microestructurales que ocurren durante la deformación superplástica. A continuación, se dará la correlación entre los cambios microestructurales surgidos y los mecanismos que podrían tener lugar.

\section{PROCEDIMIENTO EXPERIMENTAL}

Se utilizó una chapa laminada de $3 \mathrm{~mm}$ de espesor de la aleación $\mathrm{Al}-6 \% \mathrm{Cu}-0,4 \% \mathrm{Zr}$ (\% en peso).

Para la caracterización mecánica, se prepararon muestras de tracción (longitud $\times$ anchura $\times$ espesor $=10 \times 5 \times 3 \mathrm{~mm}^{3}$ ) de tal forma que el eje de tracción fuese paralelo a la dirección de laminación (DL). La caracterización microestructural se realizó llevando a cabo observaciones con el microscopio electrónico de barrido.
Las medidas de textura se realizaron mediante el análisis de rayos $\mathrm{X}$ en el modo de reflexión de Schulz empleando un difractómetro SIEMENS con goniómetro utilizando un cátodo $\mathrm{CuK} \alpha$ con un filtro de radiación $\beta$. Las figuras de polos se obtuvieron utilizando los ángulos de Euler, $\varphi 1=\quad \operatorname{rotación} / \mathrm{DN}(\mathrm{z}), \quad \Phi=$ rotación $/ \mathrm{DT}(\mathrm{y}) \quad \mathrm{y}$ $\varphi 2=$ rotación $/ \mathrm{DN}(z)$.

\section{RESULTADOS}

En la figura 1 se observa la microestructura del material en estado de recepción. La fig. 1a muestra un corte transversal del material. Se observa que la microestructura está formada por un estratificado de bandas muy estrechas paralelas a la dirección de laminación con un espesor de aproximadamente $1 \mu \mathrm{m}$. Observaciones de microscopía electrónica
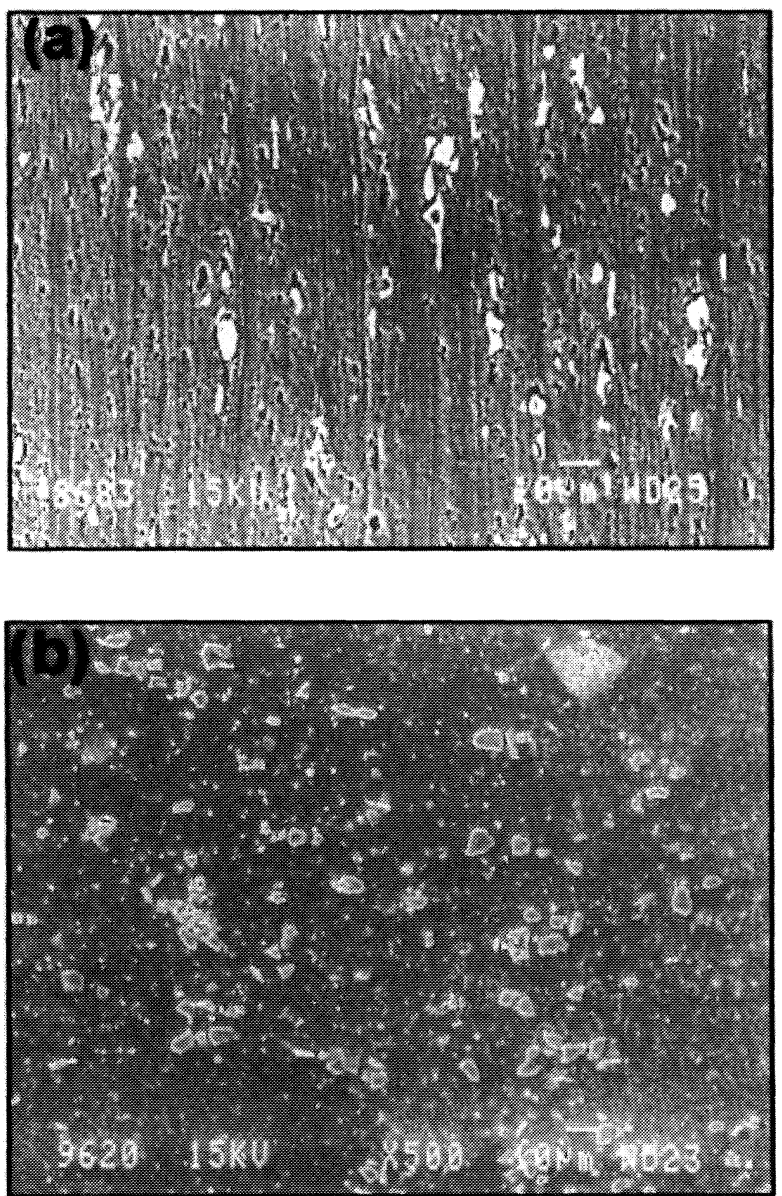

Figura 1. Microestructura de partida: a) sección transversal mostrando bandas paralelas a la $\mathrm{DL}, \mathrm{b})$ sección longitudinal mostrando partículas de $\mathrm{Al}_{2} \mathrm{Cu}$ (MEB).

Figure 1. Microstructure of the as-received material: a) cross-section view showing bands elongated towards the $R D$, b) longitudinal section showing the $\mathrm{Al}_{2} \mathrm{Cu}$ particles (SEM). $1.5 \mathrm{C}$. 
de transmisión revelaron que estas bandas encierran una subestructura muy deformada. Esquemáticamente, el material puede considerarse como un sandwich de láminas delgadas prensadas. En la figura 2(b) se revela que el tamaño de las partículas de segunda fase $\mathrm{Al}_{2} \mathrm{Cu}$ oscila entre 2 y $10 \mu \mathrm{m}$.

La figura 2 muestra figuras de polos (111) correspondientes al material de partida: (a) en la superficie de la chapa que es el plano de laminación $(\mathrm{PL})$ y $(\mathrm{b})$ en el centro de la chapa en un paralelo al PL. La textura en la superficie se interpreta como una fibra $\beta$ cuyo máximo, en el espacio de Euler, corresponde a la orientación de cobre $\{311\}<233>$ $\mathrm{C} \mathrm{Cu})$ mientras en el centro el máximo corresponde a la orientación $\{631\}<113>$ que es muy próxima a la orientación $\{421\}<211>$ (S). En las figuras de polos se observa una dispersión de orientaciones en ambas direcciones, la transversal (DT) y la normal (DN). La microestructura de partida puede representarse como compuesta por tres zonas, dos zonas superficiales similares con una subestructura orientada hacia $\{311\}<233>$ y una zona central con la orientación $\{631\}<113>$ (Fig. 3).

En la figura 4(a) se da la curva de tensión real $(\sigma)$ en función de la deformación real $(\varepsilon)$ a una

(a)

$\{311\}<233>$
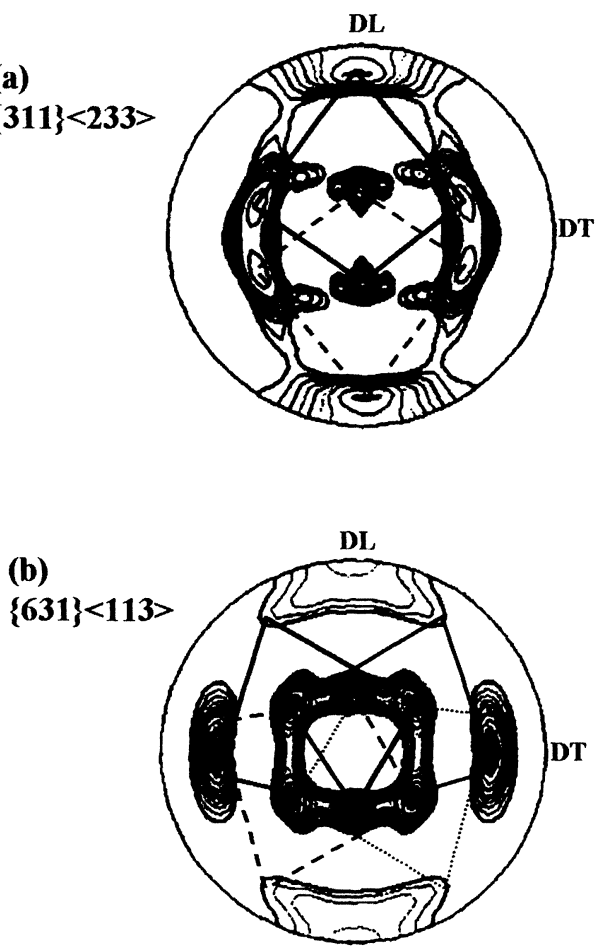

Figura 2. Figura de polos (111): a) superficie de la chapa b) centro.

Figure 2. (111) poles figure: a) surface plane, b) mid-plane of the sheet. temperatura de $480{ }^{\circ} \mathrm{C}$ y velocidad de deformación de $5 \cdot 10^{-4} \mathrm{~s}^{-1}$. La curva presenta tres estados de deformación: un estado I de endurecimiento seguido de un estado estacionario (estado II) y otro estado de endurecimiento que transcurre hasta rotura (estado III). En la misma figura se ha incorporado la curva de la sensibilidad a la velocidad de deformación, m, en función de la deformación. Se observa que el valor de m aumenta en el estado I de deformación hasta alcanzar un valor aproximado de 0,5 que se mantiene durante el estado II. Sin embargo, en el estado III decrece.

En la figura 4(b) se representa la función de distribución de orientaciones $\mathrm{f}(\mathrm{g})$ en función del alargamiento de una muestra ensayada a $480^{\circ} \mathrm{C} / 5 \cdot 10^{-4} \mathrm{~s}^{-1}$. Se observa que durante el estado

(a)
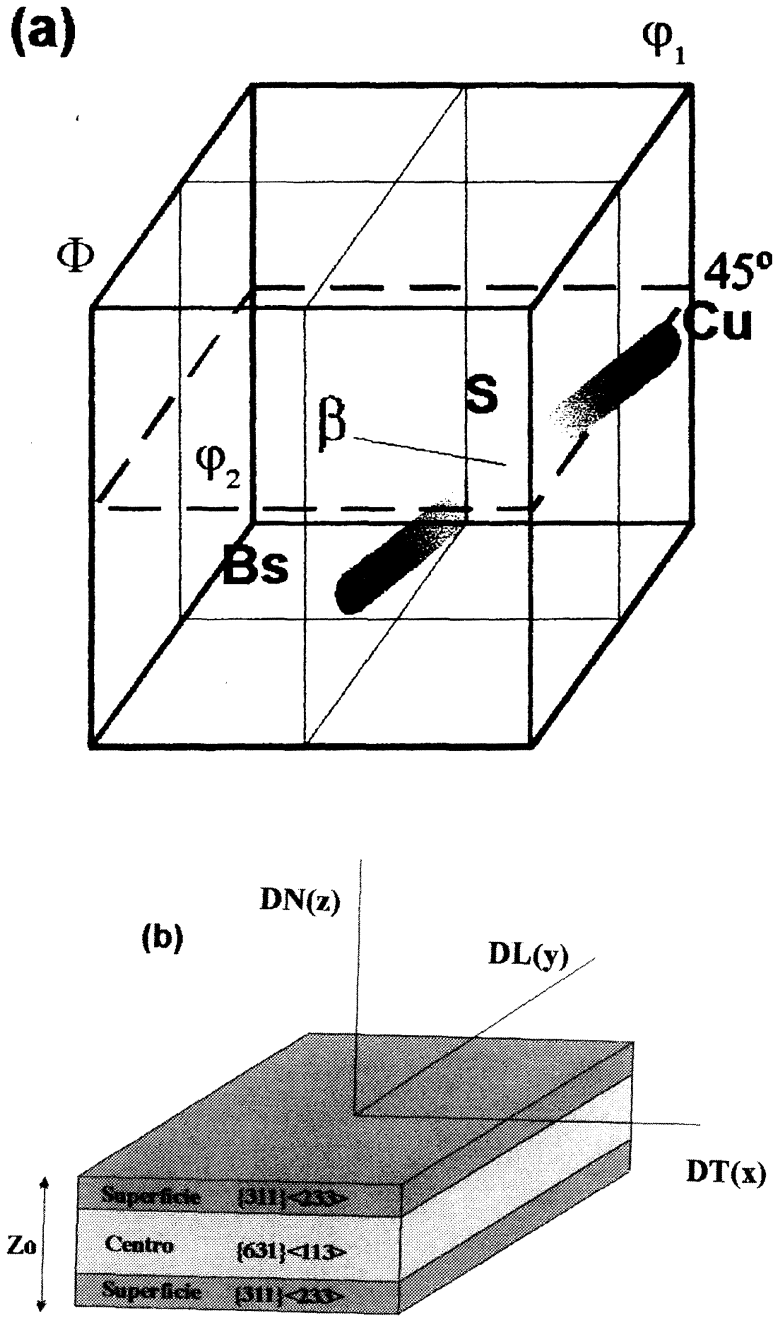

Figura 3. Esquema representativo de la textura del material de partida: a) posición de la fibra $\beta$ en el espacio de Euler, b) gradientes de textura en el estado de recepción.

Figure 3. Sketch representing the texture in the as-received state: a) position of the $\beta$-fibre orientations in the Euler space, b) gradient of texture of the as-received alloy.

Rev. Metal. Madrid 37 (2001) 

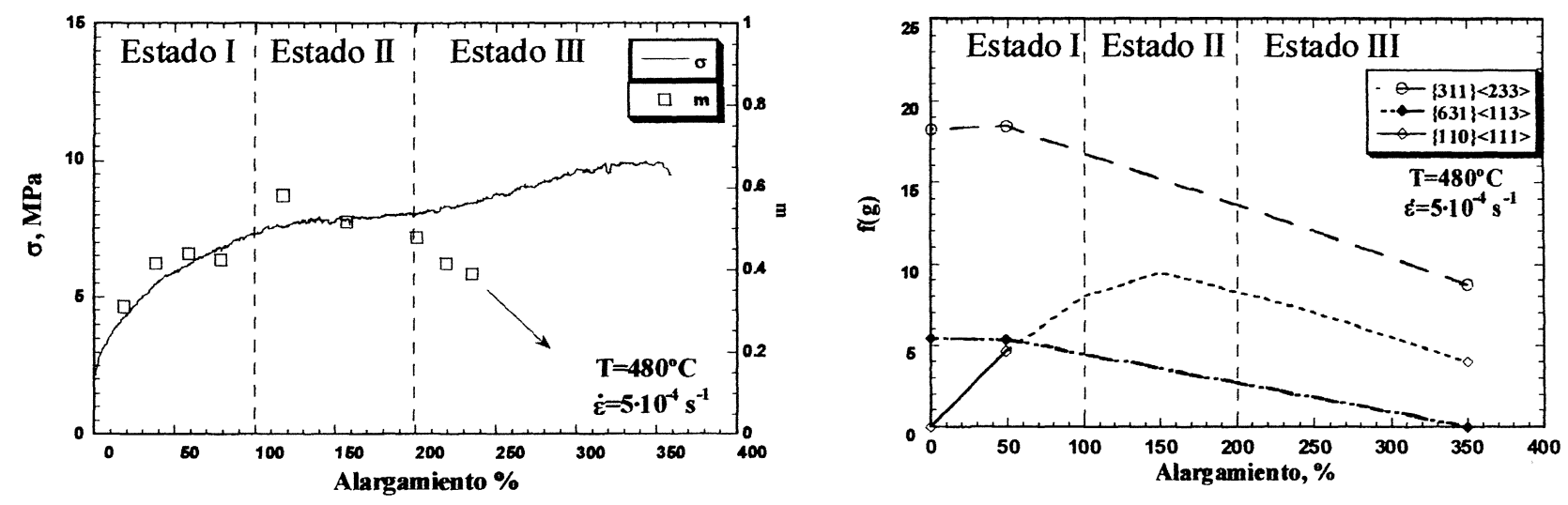

Figura 4. a) Tensión real y sensibilidad a la velocidad de deformación frente al alargamiento a $480{ }^{\circ} \mathrm{C} / 5 \times 10-4 \mathrm{~s}-1$, b) función de densidad de orientaciones en función del alargamiento.

Figure 4. a) True stress and strain rate sensitivity as a function of elongation at $480{ }^{\circ} \mathrm{C} / 5 \times 10-4 \mathrm{~s}-1$, b) orientation density functions as a function of elongation.

I de deformación la orientación $\{311\}<233>$ en la zona de superficie aumenta ligeramente. En el centro de la chapa la orientación $\{631\}<113>$ se mantiene y se observa la formación de la orientación $\{110\}<111>$. Durante el estado II y III la intensidad de las orientaciones de partida disminuyen continuamente con la deformación mientras que la orientación $\{110\}<111>$ se mantiene.

\section{DISCUSIÓN}

La formación de la orientación $\{311\}<233>$ en la superficie y la $\{631\}<133>$ en el centro de la chapa de la aleación de $\mathrm{Al}-6 \% \mathrm{Cu}-0,4 \% \mathrm{Zr}$ se debe a gradientes de orientaciones durante la preparación del material. Es de esperar que durante laminación, la deformación a través del espesor de la chapa no es homogénea y la deformación en la superficie puede ser mayor que en el centro dando lugar a gradientes de microestructura. Esquemáticamente, el material puede considerarse como un sandwich compuesto, principalmente, por tres zonas: una zona central cubierta, por ambos lados de la chapa, por una zona superficial (Fig. 3). Consecuentemente, si el material se somete a un ensayo de tracción (o de fluencia) cabe esperar que el comportamiento global del material será la combinación de los comportamientos de las tres zonas. Las heterogeneidades de microestructura (estructura de grano y textura) a través del espesor de la chapa de laminación durante el proceso termomecánico, PTM, ha sido observado en aluminio y sus aleaciones ${ }^{[9]}$. El origen de estas heterogeneidades no ha sido estudiado de manera sistemática, pero se atribuye, generalmente, a los gradientes de temperatura y de tensiones que se generan durante el PTM entre la superficie y el centro de la chapa de laminación.

Durante el estado I de la DSP a $480^{\circ} \mathrm{C} / 5 \cdot 10^{-4} \mathrm{~s}^{-1}$ ( $\varepsilon=40 \%$ ) el endurecimiento, $\mathrm{g}$, decrece y el valor de $\mathbf{m}$ aumenta. La microestructura, en paralelo, evoluciona a una estructura de (sub)granos recristalizados, por fragmentación de la microestructura inicial de bandas ${ }^{[10]}$. Esto se debe a la operación de procesos tales como la restauración y la recristali-

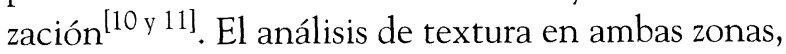
superficial y central, muestra un ligero aumento en la intensidad de las orientaciones $\{311\}<233>$ y $\{631\}<133>$, lo que confirma la operación de dichos procesos. Sin embargo, en este estado I, en la zona del centro aparece, aunque de manera suave, la orientación $\{110\}<111>$. Esto sugiere que, además de los procesos mencionados anteriormente, surgen otros procesos que tienen una naturaleza distinta, tal como puede ser el movimiento de dislocaciones. Esto puede ser evidente ya que, inicialmente, la distribución de orientaciones en las dos zonas (superficie y centro) es distinta. Por ello, es de esperar que la estimulación de los sistemas de deslizamiento en cada orientación, que viene determinada por el parámetro de Taylor o factor de Schmid, será a su vez distinta.

En el estado II de la DSP, el valor global de $\gamma$ es cero y $\mathbf{m}$ mantiene un valor constante de 0,5 . En la literatura este valor de $\mathrm{m}=0,5$ está asociado al mecanismo de deformación por deslizamiento de fronteras de grano que produce, generalmente, una disminución de textura. En el caso de la aleación Al-6\% Cu-0,4\% Zr, efectivamente, en la zona de 
superficie se observó que la intensidad de la orientación $\{311\}<233>$ decrece en el estado II sugiriendo que el DFG puede operar. Sin embargo, en la zona del centro la orientación $\{631\}<133>$ desaparece a expensas de la orientación $\{110\}<111>$. Este resultado se interpreta mediante una rotación continua de la orientación $\{631\}<133>$ durante la deformación respecto al eje $<110>/ 25^{\circ}$, lo que pone en evidencia la operación de procesos de movimiento de dislocaciones. Se deduce, por consiguiente que el comportamiento global del material es la combinación de ambos comportamientos, el DFG en la superficie más el movimiento de dislocaciones en el centro. Consecuentemente, el valor global de $\mathbf{m}$, por si solo, no es suficiente para determinar el mecanismo verdadero responsable del comportamiento microestructural del material.

En el estado III, el valor global de $\gamma$ es distinto de cero y $\mathbf{m}$ decrece. Globalmente, la microestructura está alargada en la dirección de tracción, lo que sugiere que el proceso de movimiento de dislocaciones en el centro prevalece sobre el DFG en la superficie. La textura determinada en este estado II confirma estas observaciones. La orientación $\{311\}<233>$ tiende a desaparecer y la $\{631\}<113>$ desaparece totalmente transformándose en la orientación $\{110\}<111>$.

\section{CONCLUSIONES}

- La aleación Al-6\% Cu-0,4\% Zr en estado de recepción presenta heterogeneidades de textura a través del espesor de la chapa de laminación. La zona de la superficie se caracteriza por la orientación $\{311\}<233>$ y la del centro por la orientación $\{631\}<113>$.

- Durante la deformación a $480{ }^{\circ} \mathrm{C} / 5 \cdot 10^{-4} \mathrm{~s}^{-1}$ se observaron tres estados de deformación:

i) En el estado I el endurecimiento g decrece y la sensibilidad a la velocidad de deformación $\mathbf{m}$ aumenta hasta alcanzar el valor 0,5 (inicio del estado II). La microestructrua de bandas se fragmenta en (sub)granos recristalizados. Globalmente, la textura inicial aumenta ligeramente, debido a procesos de recristalización.

ii) Durante el estado II el valor de g es cero y $\mathbf{m}$ es constante. La textura en la superficie decrece mientras que en el centro se forma la $\{110\}<111>$. Se sugiere que la combinación de DFG en la superficie con el movimiento de dislocaciones en el centro es el mecanismo responsable de la deformación.

iii) En el estado III, $g$ es distinto de cero y $\mathbf{m}$ decrece. La textura en la superficie decrece y, en el centro, la orientación $\{110\}<111>$ se mantiene a expensas de la desaparición de la orientación $\{631\}<113>$.

\section{REFERENCIAS}

[1] F. Barlat, J.C. Brem y J. Liu, Scripta Metall. Mater. 27 (1992) 1121-1126.

[2] A.K. Vasudévan, W.G. Fricke, Jr., R.C. Malcolm, R.J. Bucci, M.A. Przystupa y F. Barlat, Metall. Trans. 19A (1988) 731-732.

[3] M. Peters, J. Eschweiler y K. Welpmann, Scripta Metall. 20 (1986) 259-264.

[4] P.L. Blackwell y P.S. Bate, Metall. Trans. 24 (1993) 1085-1093.

[5] B.M. Watts y M.J. Stowell, J. Mater. Sci. 6 (1971) 228237.

[6] B.M. Watts, M.J. Stowell, B.L. Baikie y D.G.E. Owen, Met. Sci. 10 (1976) 189-197.

[7] K. Matsuki, K. Minami, M. Tokizawa y Y. Murakami, Met. Sci. 13 (1979) 619-626.

[8] R.H. Bricknell y W. Edington, Acta Metall. 27 (1979) 1313-1318.

[9] W. Truszkowski, J. KRól y B. Major, Metall. Trans. 11 (1980) 749-758

[10] M. Eddahbi, Tesis Doctoral, Facultad de Ciencias Físicas, Universidad Complutense de Madrid, 1998.

[11] Gudmudsson, D. Brooks y J.A. Wert, Acta Metall. 39 (1991) 19-35. 